\title{
Harvesting microalgal-bacterial biomass from biogas upgrading process and evaluating the impact of flocculants on their growth during repeated recycling of the spent medium
}

\author{
María del Rosario Rodero ${ }^{\mathrm{a}, \mathrm{b}, \mathrm{d}}$, Raúl Muñoz ${ }^{\mathrm{a}, \mathrm{b}}$, Raquel Lebrero ${ }^{\mathrm{a}, \mathrm{b}}$, An Verfaillie ${ }^{\mathrm{c}, \mathrm{d}}$, Jonas Blockx ${ }^{\mathrm{c}, \mathrm{d}}$, \\ Wim Thielemans $^{c}$, Koenraad Muylaert ${ }^{\mathrm{d}}$, RamasamyPraveenkumar ${ }^{\text {c,d,e,** }}$ \\ ${ }^{a}$ Department of Chemical Engineering and Environmental Technology, University of Valladolid, Dr. Mergelina s/n., 47011 Valladolid, Spain \\ ${ }^{\mathrm{b}}$ Institute of Sustainable Processes, University of Valladolid, 47011 Valladolid, Spain \\ c Sustainable Materials Lab, KU Leuven, Campus Kulak Kortrijk, Etienne Sabbelaan 53, box 7659, B-8500 Kortrijk, Belgium \\ d Laboratory for Aquatic Biology, KU Leuven, Campus Kulak Kortrijk, Etienne Sabbelaan 53, box 7659, B-8500 Kortrijk, Belgium \\ e Environmental Dynamics, Department of Science and Environment, Roskilde University, Universitetsvej 1, 4000 Roskilde, Denmark
}

\section{A R T I C L E I N F O}

\section{Keywords}

Microalgae

Harvesting

Flocculation

Cellulose nanocrystals

Zetag

Screening

\begin{abstract}
A B S T R A C T
Microalgal-bacterial consortium can be used to upgrade biogas by removing $\mathrm{CO}_{2}$ and $\mathrm{H}_{2} \mathrm{~S}$. Photosynthetic biogas upgrading requires harvesting microalgal-bacterial biomass in order to use the biomass-free cultivation medium as scrubbing liquid in the absorption column. In this study, the efficiency of different flocculants (Zetag 8125, cationically modified cellulose nanocrystals, Tanfloc, chitosan, and $\mathrm{FeCl}_{3}$ ) to harvest microalgal-bacterial biomass used for biogas upgrading in alkaline medium (inorganic carbon concentration up to $1800 \mathrm{mg} \mathrm{L}^{-1}$ and a pH $\sim 10$ ) was evaluated. Zetag and cationic cellulose nanocrystals resulted in maximum flocculation efficiencies of $95 \%$ (optimal dose $30 \mathrm{mg} \mathrm{g}^{-1}$ ) and 93\% (optimal dose $20 \mathrm{mg} \mathrm{g}^{-1}$ ), respectively. Low flocculation was observed with other flocculants at doses as high as $200 \mathrm{mg} \mathrm{g}^{-1}$, which can be ascribed to the high $\mathrm{pH}$ of the alkaline medium. Zetag and cationic cellulose nanocrystals were selected for harvesting the biomass during semi-continuous cultivation of the microalgal consortium. Both Zetag and cationic cellulose nanocrystals were effective in flocculating the biomass with efficiencies of over $90 \%$ during five successive harvesting cycles. Gravity settling of the flocs formed by Zetag and cationic cellulose nanocrystals resulted in low biomass concentration factors of 7.7 and 2.0, respectively. Screening of flocs using a nylon mesh screen (pore size of $180 \mu \mathrm{m}$ ) resulted in a biomass concentration factor as high as 19.8. Zetag and cationic cellulose nanocrystals could be useful in harvesting biomass under high alkaline conditions without detrimental effects on biomass growth.
\end{abstract}

\section{Introduction}

Biogas from the anaerobic digestion of organic waste or wastewater constitutes a promising renewable energy vector able to reduce our current dependence on fossil fuels due to its high $\mathrm{CH}_{4}$ content (40-75\%) [1]. In this context, the removal of biogas pollutants, mainly $\mathrm{CO}_{2}$ and $\mathrm{H}_{2} \mathrm{~S}$, is a mandatory step for its use as a natural gas substitute [2]. Photosynthetic biogas upgrading in high-rate algal ponds coupled with an external absorption column has recently emerged as a low cost (energy consumption of $0.08 \mathrm{~kW}-\mathrm{h}\left(\mathrm{Nm}^{3} \text { treated biogas }\right)^{-1}$ ) and environmentally friendly $\left(\mathrm{CO}_{2}\right.$ emissions of $\left.21 \mathrm{~g}-\mathrm{CO}_{2}\left(\mathrm{Nm}^{3} \text { treated biogas }\right)^{-1}\right)$ alternative to conventional physical-chemical technologies to remove $\mathrm{CO}_{2}$ and $\mathrm{H}_{2} \mathrm{~S}$ from biogas (energy consumption and $\mathrm{CO}_{2}$ emissions of $0.30 \mathrm{kWh}$ and $944 \mathrm{~g}-\mathrm{CO}_{2}$ to obtain $1 \mathrm{Nm}^{3}$ of treated biogas, respectively, for an activated carbon filter combined with a water scrubbing) [3]. Main- taining a high alkalinity (inorganic carbon concentration $>1500 \mathrm{mg} \mathrm{L}^{-1}$ ) and $\mathrm{pH} \sim 10$ of the cultivation medium is essential to increase the mass transfer of acidic gases like $\mathrm{CO}_{2}$ and $\mathrm{H}_{2} \mathrm{~S}$ from the biogas to the cultivation medium [4]. Hence, the use of alkaliphilic microalgal-bacterial consortia able to withstand high inorganic carbon concentrations is essential to efficiently remove $\mathrm{CO}_{2}$ and $\mathrm{H}_{2} \mathrm{~S}$ from the cultivation medium in high-rate algal ponds [5]. The biogas upgrading process is based on the use of part of the biomass-free cultivation medium as scrubbing liquid in the absorption column. In this sense, separating the microalgal-bacterial biomass generated in high-rate algal ponds from the scrubbing liquid constitutes a critical step. It also allows for control over microalgal productivity under operation with no effluent as a consequence of evaporation losses of water when using digestate as nutrient source (due to its high nutrient concentration,

\footnotetext{
* Corresponding author at: Environmental Dynamics, Department of Science and Environment, Roskilde University, Universitetsvej 1, 4000 Roskilde, Denmark.

E-mail address: praveen@ruc.dk
} 
which consequently requires low digestate flowrates to sustain algal-bacterial growth) [6].

Several microalgae harvesting methods such as centrifugation, flotation, sedimentation, or filtration have been reported [7]. However, due to low biomass concentration of microalgae in high-rate algal ponds (0.2-1.2 $\left.\mathrm{g} \mathrm{L}^{-1}\right)$ and their small cell size (typically in micrometers), some of these technologies do not achieve an efficient solid-liquid separation or they are limited by high-energy requirements with associated increases in operational costs $[8,9]$. In this regard, flocculation followed by a solid-liquid separation step, such as gravity sedimentation or screening, is considered a rapid and cost-effective alternative for a large-scale harvesting of microalgal biomass [10]. During flocculation, the addition of chemicals leads to the aggregation of microalgal cells forming large flocs [11]. Flocculation can be induced by neutralizing the surface charge of the cells (charge neutralization), by partially reversing the charge of the particle surface, resulting in the connection of particles through patches with opposite charge (electrostatic patch), by precipitation caused by an aggregating polymer network that entangles microalgal cells (sweeping mechanism), or by forming bridges between individual particles (bridging) $[12,13]$.

The optimal dose of the flocculants depends on the characteristics of the microalgal species (i.e. cell size, culture age, and cell wall composition) and the flocculant (e.g. charge, rigidity, and morphology) [14]. Inorganic salts, such as $\mathrm{FeCl}_{3}$, which induce flocculation via charge neutralization, have been widely used as flocculants due to their low cost, in spite of needing higher dose compared to other flocculants $[15,16]$. Organic polymers such as Zetag, a synthetic copolymer of acrylamide and quaternized cationic monomers, which are able to interact with microalgal cells by charge neutralization and bridging, have been successfully applied in the flocculation of various microalgae $[17,18]$.

Flocculants based on natural biopolymers are attracting interest as flocculants due to their biodegradability. Chitosan from chitin waste is a non-toxic and inexpensive biopolymer composed of linear poly-amino-saccharide chains that can agglomerate individual cells through different mechanisms such as charge neutralization, bridging, sweeping, and adsorption [19-21]. Tanfloc is a commercial biopolymer based on tannins extracted from bark of Acacia mearnsii that has also been used as a flocculant for microalgae $[18,22]$. More recently, cationically modified cellulose nanocrystals (CNCs) have been introduced as a flocculant for microalgae [23-26]. CNCs have a high aspect ratio and high external surface area $\left(\sim 300 \mathrm{~m}^{2} \mathrm{~g}^{-1}\right)$, which is favorable for flocculation. Moreover, they can be readily modified by addition of a wide range of polymer matrices to obtain a flocculant with desired surface characteristics $[27,28]$.

The $\mathrm{pH}$ of the culture medium is one of the crucial factors for the performance of the flocculants. Many flocculants get protonated and become cationic only at low $\mathrm{pH}(<7)$ [29]. In an alkaline medium, flocculants that carry a pH-independent cationic charge should have a superior performance. Many polymer flocculants experience coiling in high ionic strength conditions and are expected to perform poorly in a medium with a high inorganic carbon concentration [30,31]. Hence, the selection of a flocculant that functions at high $\mathrm{pH}$ and at high inorganic carbon concentration is essential for photosynthetic biogas upgrading. Another important feature while applying flocculants in biogas upgrading systems is to obtain a biomass-free medium that can be repeatedly recycled without any detrimental effect on the growth of microalgae and bacteria. Recycling of the spent medium from the absorption column to the photobioreactor is essential for the subsequent removal of $\mathrm{CO}_{2}$ and $\mathrm{H}_{2} \mathrm{~S}$ from the medium. While $\mathrm{CO}_{2}$ will be consumed by microalgae, $\mathrm{H}_{2} \mathrm{~S}$ will be oxidized to sulphate by sulphur oxidizing bacteria using the oxygen that is generated photosynthetically [32]. In this regard, it is important that accumulation of the flocculant and/or algal organic matter in the recycled culture medium should not lead to microalgal-bacterial growth inhibition $[33,34]$. Furthermore, the flocculant needs to be versatile in harvesting altogether different microalgal species present in the consortium. Otherwise, those species of microalgae that did not flocculate would eventually alter the microalgal community structure and ultimately make the flocculation process inefficient. So far, no studies have focused on the selection of a suitable flocculant and its dose for efficient use in a repeated recycling of cultivation medium, in spite of the crucial role of this separation step in photosynthetic biogas upgrading.

The aim of this study was to optimize harvesting of a microalgal-bacterial consortium using flocculation, followed by a solid-liquid separation for a photosynthetic biogas upgrading process which requires working under high $\mathrm{pH}(\sim 10)$ and alkalinity (inorganic carbon concentration up to $1800 \mathrm{mg} \mathrm{L}^{-1}$ ), and to evaluate the effect of flocculants on the biomass while recycling the culture medium. For this purpose, different flocculants such as, Zetag® 8125 , cationic CNCs, Tanfloc, chitosan, and $\mathrm{FeCl}_{3}$ were tested. Furthermore, the recyclability of the medium after flocculation for the effective flocculants (Zetag and cationic CNCs) was evaluated in a semi-continuous cultivation system. Finally, the feasibility of using screening instead of gravity settling to separate biomass flocs from the culture medium was also assessed.

\section{Materials and methods \\ 2.1. Cultivation of microalgal-bacterial consortium}

Microalgal-bacterial consortium was obtained from an indoor high-rate algal pond used for biogas upgrading using a high alkalinity synthetic medium as nutrient source located at the Department of Chemical Engineering and Environmental Technology at University of Valladolid. The consortium was grown in $2 \mathrm{~L}$ bottles (diameter: $136 \mathrm{~mm}$, working volume: $1.5 \mathrm{~L}$ ) as fed-batch cultures in a synthetic medium composed of $\left(\mathrm{g} \mathrm{L}^{-1}\right): 7.60 \mathrm{NaHCO}_{3}, 3.70 \mathrm{Na}_{2} \mathrm{CO}_{3}, 0.58 \mathrm{~K}_{2} \mathrm{HPO}_{4}, 1.91$ $\mathrm{NH}_{4} \mathrm{Cl}, 0.10 \mathrm{MgSO}_{4} \cdot 7 \mathrm{H}_{2} \mathrm{O}, 0.02 \mathrm{CaCl}_{2} \cdot 2 \mathrm{H}_{2} \mathrm{O}$ and $1 \mathrm{~mL}$ of a trace metal solution prepared according to the Wright's cryptophyte medium [35]. The cultivation medium was maintained at $\mathrm{pH} \sim 10$ and fed with $25 \mathrm{~mL}$ of fresh medium every day, based on the data on the hydraulic retention time used in the high rate algal pond for biogas upgrading [36]. The flasks were aerated by bubbling with $0.2-\mu \mathrm{m}$ filtered air and mixed using magnetic stirrers. Cultures were continuously illuminated from front and backside of the flask, each at an intensity of $\sim 100 \mu \mathrm{mol} \mathrm{m}^{-2} \mathrm{~s}^{-1}$ and maintained at $24^{\circ} \mathrm{C}$ in a temperature-controlled room.

\subsection{Selection of optimal flocculants for use in alkaline and high $\mathrm{pH}$ conditions}

Flocculation efficiencies of five flocculants: Zetag® 8125 (BASF, Germany, hereinafter referred as Zetag), in-house developed CNCs grafted with methylimidazolium cationic group (MIM-g-CNCs) [25], $\mathrm{FeCl}_{3} \cdot 6 \mathrm{H}_{2} \mathrm{O}$ (Chem-lab, >99\%), Tanfloc ${ }^{\circledR}$ SG (Tanac, Brazil), and chitosan (Sigma-Aldrich 417963) were tested on the microalgal-bacterial consortium using standard jar tests. For each flocculant a stock solution of $5 \mathrm{~g} \mathrm{~L}^{-1}$ was prepared in distilled water. The stock solution of chitosan $\left(5 \mathrm{~g} \mathrm{~L}^{-1}\right.$ ) was prepared in a $0.04 \mathrm{M} \mathrm{HCl}$ solution due to its slow dissolution in distilled water [20].

To evaluate harvesting of microalgae-bacterial biomass using different flocculants, conditions for the jar test such as initial stirring speed (300-900 rpm), stirring time (5-30 min), floc settling time (15-120 min), and biomass concentration $\left(0.2-2 \mathrm{~g} \mathrm{~L}^{-1}\right)$ were initially optimized with $30 \mathrm{mg} \mathrm{g}^{-1}$ of Zetag or MIM- $\mathrm{g}$-CNCs in order to achieve optimal flocculation efficiency and biomass concentration factor (Supplementary material, Fig. S1).

Dose-response curves for the flocculants were determined by adding different concentrations of flocculants (ranging from 0 to $200 \mathrm{mg} \mathrm{g}^{-1}$ 
) to $50 \mathrm{~mL}$ of microalgae-bacteria suspension ( $\left.\sim \mathrm{g} \mathrm{L}^{-1} \mathrm{TSS}\right)$ while vigorously mixing at $700 \mathrm{rpm}$ with a magnetic stirrer. Following the addition of flocculants, the suspension was gently mixed at $200 \mathrm{rpm}$ for $5 \mathrm{~min}$ to promote flocculation. After this, the suspension was decanted in $50 \mathrm{~mL}$ plastic tubes and the flocs were allowed to settle for $60 \mathrm{~min}$ before measuring the volume and the optical density $(750 \mathrm{~nm})$ of the supernatant (Genesis 10S UV-Vis; Thermo Fisher, US). The flocculation efficiency $\left(\eta_{a}\right)$ was calculated based on measurement of the optical density before flocculants addition $\left(\mathrm{OD}_{\mathrm{i}}\right)$ and of the supernatant after settling $\left(\mathrm{OD}_{\mathrm{f}}\right)$ according to the following equation:

$\eta_{a}(\%)=\frac{O D_{i}-O D_{f}}{O D_{i}} \times 100$

In addition, the biomass concentration factor was calculated as:

$C F=\frac{C_{f}}{C_{i}}$

where $\mathrm{C}_{\mathrm{i}}$ and $\mathrm{C}_{\mathrm{f}}$ were the initial biomass concentration before addition of flocculants and final biomass concentration in the volume containing the flocculated microalgae, respectively. The jar tests were carried out in duplicate and the results were represented as the average values along with their corresponding standard deviation.

\subsection{Repeated recycling of spent medium}

Based on the performance of the flocculants, Zetag and MIM-g-CNCs were chosen for experiments with repeated recycling of the spent medium in order to check the effectiveness of the flocculants in a semi-continuous cultivation system. In these experiments, three $2 \mathrm{~L}$ bottles (working volume $1.5 \mathrm{~L}$ ) with synthetic medium were inoculated with the microalgal-bacterial consortium (initial biomass concentration of $0.2 \mathrm{~g} \mathrm{~L}^{-1}$ ) and incubated under similar conditions as described in Section 2.1. Following 4 days of incubation, $500 \mathrm{~mL}$ of the culture from each bottle were harvested either by centrifugation or by Zetag or MIM-g-CNCs-based flocculation, and the spent medium was recycled to the culture bottles. The working volume of the cultures was maintained at $1.5 \mathrm{~L}$ by addition of fresh medium $\left(\mathrm{NH}_{4}{ }^{+}\right.$concentration of $100 \mathrm{mg} \mathrm{L}^{-1}$ to avoid ammonia inhibition) after harvesting in order to compensate losses in the spent medium. The harvesting of the control cultures was performed by centrifugation at $6000 \mathrm{rpm}$ for $10 \mathrm{~min}$ following 30 min settling to test autoflocculation. For Zetag or MIM- $g$-CNCs -based flocculation, the suspensions in a beaker were mixed intensively (250 rpm) with an overhead stirrer for $1 \mathrm{~min}$ following the addition of the flocculant. Then, the suspensions were gently mixed $(50 \mathrm{rpm})$ for another $20 \mathrm{~min}$, after which they were allowed to settle for $30 \mathrm{~min}$ in a $500 \mathrm{~mL}$ Imhoff cone. The recycling experiments were repeated for $5 \mathrm{cy}-$ cles during 14 days with doses for Zetag and MIM- $g$-CNCs ranging from 25-49 and 20-40 $\mathrm{mg} \mathrm{L}^{-1}$, respectively.

The specific growth rate $(\mu)$ was calculated as:

$\mu=\frac{\ln \left(\frac{c_{2}}{c_{1}}\right)}{t_{2}-t_{1}}$

where $c_{1}$ and $c_{2}$ were the biomass concentration at times $t_{1}$ and $t_{2}$.

The biomass concentration was measured as total suspended solids (TSS; $\mathrm{g} \mathrm{L}^{-1}$ ). TSS was determined gravimetrically based on GF/C filtration (Whatman, UK) and drying of biomass at $105^{\circ} \mathrm{C}$ overnight after washing them 2-3 times with distilled water in order to remove the inorganic salt residue [37]. A linear correlation of optical density values of the culture at $750 \mathrm{~nm}$ against TSS (TSS g L ${ }^{-1}=0.7234 \times \mathrm{OD}_{750}$ $\mathrm{nm}-0.0699$ ) was obtained. The $\mathrm{pH}$ of the culture medium was monitored every day (Consort C1010; Consort bvba, Belgium) and adjusted to $\sim 10$ before the harvesting by adding the necessary volume of $2 \mathrm{M}$
$\mathrm{HCl}$ solution. $\zeta$-Potential of the cultivation medium was measured (NanoBrook Omni; Brookhaven Instruments, US) in triplicate before and after flocculation to monitor the flocculant accumulation in the spent medium and the results were represented as the average values along with their corresponding standard deviation. The inorganic carbon concentration was measured before flocculation using a carbonate hardness test (Merck Millipore, Germany).

\subsection{Separation of flocs by gravity sedimentation and screening}

Screening using a nylon mesh screen with pore size of $180 \mu \mathrm{m}$ (Elko filtering Co., Switzerland) was evaluated for solid-liquid separation following flocculation to increase the concentration factor. Biomass was flocculated with either Zetag (20 $\mathrm{mg} \mathrm{g}^{-1}$ ) or MIM- $g$-CNCs $\left(40 \mathrm{mg} \mathrm{g}^{-1}\right.$ ) and allowed to settle for $30 \mathrm{~min}$. Following settling, the entire volume of the suspension was screened through the nylon mesh screen. The flocculation efficiency and the concentration factor were calculated as described in Section 2.2. These experiments were carried out in duplicate and the results were represented as the average values along with their corresponding standard deviation.

\section{Results and discussion}

\subsection{Flocculation of microalgal-bacterial biomass from fed-batch cultures}

The microalgal-bacterial consortium was mainly composed of Chlorella sp., Oscillatoria spp., and uncharacterized bacterial species. Microscopic observation at different time points of fed-batch cultivation confirmed the stable composition of the microalgal consortium.

Among the five different flocculants tested, Zetag and MIM-g-CNCs resulted in efficient flocculation of the microalgal-bacterial consortium. While Zetag triggered a maximum flocculation efficiency of $95 \%$ with a dose of $30 \mathrm{mg} \mathrm{g}^{-1}$ ( $\mathrm{g}$ flocculant $\mathrm{g}^{-1}$ dry matter biomass concentration), MIM-g-CNCs resulted in a flocculation efficiency of $93 \%$ with $20 \mathrm{mg} \mathrm{g}^{-1}$ (Fig. 1). Both are cationic polymeric flocculants carrying respectively quaternary ammonium and methyl imidazolium groups, i.e. cationic charges that are stable over a very wide $\mathrm{pH}$ range. Other synthetic cationic polymers have been reported for harvesting marine microalgae, such as Zetag 7557 and Synthofloc 5080H to harvest Phaeodactylum tricornutum and Neochloris oleoabundans at a pH 7.5 [17], and Magnafloc to harvest Chaetoceros calcitrans at a pH 10.2 [38]. With freshwater microalgae C. vulgaris, flocculation efficiency of $99 \%$ was reported with Zetag 8125 with a dose of $6.4 \mathrm{mg} \mathrm{g}^{-1}$, whereas, with marine microalgae Nannochloropsis oculata a flocculation efficiency of $\sim 44 \%$ with a dose of $155 \mathrm{mg} \mathrm{g}^{-1}$ was reported [18]. In spite of the high $\mathrm{pH} \quad(\sim 10)$ and high inorganic carbon concentra-

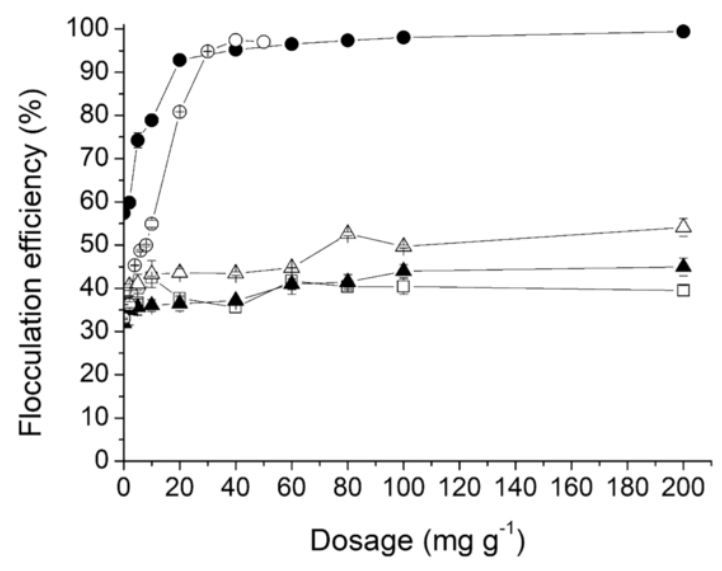

Fig. 1. Flocculation dose-response curves (average values and standard deviation; $n=2$ ) of Zetag (O), cationic cellulose nanocrystals $(\bullet), \mathrm{FeCl}_{3}(\Delta)$, Tanfloc $(\mathbf{\Delta})$ and Chitosan $(\square)$. 
tion $\left(\sim 1800 \mathrm{mg} \mathrm{L}^{-1}\right)$, a superior flocculation efficiency (95\% with $30 \mathrm{mg} \mathrm{g}^{-1}$ ) was achieved with Zetag 8125 in this study when compared to the flocculation of Nannochloropsis oculata. This could be attributed to the relatively low ion concentration in the alkaline medium used in this study compared to the marine culture medium.

In this study, in addition to Zetag, the efficiency of the methyl imidazolium-modified natural cellulose in the form of ribbon-like nanocrystals to harvest microalgal-bacterial consortium at high $\mathrm{pH}(\sim 10)$ and inorganic carbon concentrations (up to $1800 \mathrm{mg} \mathrm{L}^{-1}$ ) was demonstrated. Verfaillie et al. [26] reported a slight decrease in the flocculation efficiency (from $96 \%$ to $87 \%$ ) with the increase of salinity from 0 to $50 \mathrm{~g} \mathrm{~L}^{-1}$ when using $20 \mathrm{mg} \mathrm{L}^{-1}$ of cationic CNCs to harvest Nannochloropsis oculata. With freshwater microalgae C. vulgaris, Blockx et al. [25] reported flocculation efficiencies $>80 \%$ with $50 \mathrm{mg} \mathrm{L}^{-1}$ cationic $\mathrm{CNCs}$ at a pH 6 and a biomass concentration of $0.28 \mathrm{~g} \mathrm{~L}^{-1}$. Reportedly, cationically modified CNCs are efficient and versatile in the sense that they could be used to flocculate microalgae grown under a wide range of cultivation conditions due to their $\mathrm{pH}$ independent charge, crystalline nature that provides rigidity to avoid coiling of the polymer under high ionic strength medium, and finally, a high surface cationic charge density that results in high flocculation efficiency at low doses $[25,26]$.

Other flocculants such as $\mathrm{FeCl}_{3}$, Tanfloc, and chitosan resulted in low flocculation efficiencies (maximum values of $54 \pm 2,45 \pm 2$ and $43 \pm 0 \%$, respectively) for doses up to $200 \mathrm{mg} \mathrm{g}^{-1}$ (Fig. 1). When compared to organic polymers, inorganic salts such as ferric chloride often requires higher doses to promote flocculation [39]. However, doses higher than $200 \mathrm{mg} \mathrm{g}^{-1}$ could result in toxicity of the medium and, moreover, the presence of residual metal ions in the harvested biomass could pose problems during downstream processing [40].

Although Tanfloc has been demonstrated to flocculate marine microalgae [29], low flocculation was observed in this study as a consequence of the high pH ( $\sim 10)$ of the medium. Likewise, Selesu et al. [41] achieved a flocculation efficiency of only $30 \%$ using Tanfloc for harvesting microalgae Scenedesmus sp. at $\mathrm{pH} 11$. Having a point of zero charge of 8.17, Tanfloc assumes a neutral surface charge at higher $\mathrm{pH}$ and, consequently, loses its ability to flocculate either through charge neutralization or bridging [29]. Similarly, the conditions of the culture medium did not favor biomass flocculation using chitosan. At $\mathrm{pH}>8$, the amine groups on the surface of chitosan get deprotonated, which makes it impossible for chitosan to neutralize the microalgal surface charges to induce flocculation by charge neutralization or bridging. Moreover, the high ionic strength of the medium would result in coiling of the polymer [42,43]. Blockx et al. [20] reported that chitosan can also induce flocculation of microalgae at high $\mathrm{pH}(>7.5)$ and in seawater medium, but in that case flocculation occurs via sweeping mechanism and much higher doses of chitosan are needed than in freshwater conditions ( $>75 \mathrm{mg} \mathrm{L}^{-1}$ ). Similarly, Farid et al. [21] reported higher flocculation efficiencies of chitosan at high $\mathrm{pH}$ (9) when compared to neutral $\mathrm{pH}$ (7) with marine microalgae Nannochloropsis sp. However, no sweeping mechanism was observed in this study with chitosan doses up to $200 \mathrm{mg} \mathrm{g}^{-1}$.

Another important parameter in flocculation is the biomass concentration factor. Less concentrated biomass flocs will require a secondary dewatering process. Maximizing the quantity of culture medium that can be recycled and managing lower volumes of biomass is essential in terms of process economics [44]. Flocculation with Zetag resulted in a maximum biomass concentration factor of 6.5 at a dose of $40 \mathrm{mg} \mathrm{g}^{-1}$, while flocculation with MIM- $g$-CNCs exhibited a concentration factor of only 3.8 at a similar dose (Supplementary material, Fig. S2). Biomass concentration factors in the range of 3.5-14.1 have been reported for different cationic polymers while harvesting marine microalgae by flocculation followed by 2 hour gravity settling [17]. However, concentra- tion factors obtained in this study were less than those reported by Eyley et al. [24] who achieved concentration factor as high as 49 with freshwater microalgae $C$. vulgaris, harvesting by cationic CNCs-based flocculation and $30 \mathrm{~min}$ of gravity settling.

\subsection{Flocculation during semi-continuous cultivation and repeated recycling of spent medium}

In a photosynthetic biogas upgrading process, the spent medium after biomass harvesting is recycled to the photobioreactor through an absorption column to remove the $\mathrm{CO}_{2}$ and $\mathrm{H}_{2} \mathrm{~S}$ from the biogas. In this context, it is important to evaluate the impact of flocculation on biomass growth after recycling. Based on the previous results of this study, Zetag and MIM- $g$-CNCs were selected to study their effect during repeated recycling of spent medium. The impact of these flocculants on biomass growth was compared with that of centrifugation.

Spontaneous settling of microalgal-bacterial biomass (after $30 \mathrm{~min}$ ) without flocculants was negligible, ranging between 1 and $8 \%$ over all harvesting cycles tested. Addition of Zetag and MIM-g-CNCs resulted in maximum flocculation efficiencies of $\sim 97 \%$ at a dose of $23 \mathrm{mg} \mathrm{g}^{-1}$ and $\sim 98 \%$ at $39 \mathrm{mg} \mathrm{g}^{-1}$, respectively. Different flocculant doses were tested in the subsequent harvesting cycles in order to determine the minimum dose of flocculant. Flocculation with Zetag resulted in a flocculation efficiency of $97 \%$ with doses as low as $22 \mathrm{mg} \mathrm{g}^{-1}$, whereas, with MIM-g-CNCs, a dose of $20 \mathrm{mg} \mathrm{g}^{-1}$ only achieved $55 \%$ of flocculation (Fig. 2).

A steady growth of microalgal-bacterial biomass was observed during semi-continuous cultivation using all three harvesting methods (centrifugation, Zetag, and MIM-g-CNCs-based flocculation), over 5 cycles of repeated recycling of $500 \mathrm{~mL}$ culture medium. Harvesting by centrifugation resulted in a 5-9\% increased biomass growth when compared to flocculation-based harvesting (Fig. 2). Specific growth rates differed between the different harvesting treatments and along the time course of cultivation (Fig. S3, Supplementary material). Zetag being a synthetic polyacrylamide polymer and MIM-g-CNCs possessing an aromatically dislocated positive charge could be toxic to microalgae at high concentrations. In this regard, although slightly lower growth rates were observed in the last harvesting cycles using Zetag and MIM-g-CNCs in comparison with harvesting based on centrifugation, no detrimental effect on microalgae growth was observed along the 5 cycles. Moreover, concentrations of these flocculants were optimized to minimize the dose required to induce flocculation and to avoid the presence of free polymers in the recycled medium. This was verified through $\zeta$-potential analysis of cell free supernatant before and after harvesting at each cycle (Supplementary material, Table S4). The presence of free flocculant in the spent medium should be evident from an increase in $\zeta$-potential in the spent medium. In this study, no significant change in the $\zeta$-potential of the spent medium was observed between centrifugation, Zetag, and MIM-g-CNCs-based flocculation, demonstrating that the quantity of flocculant that was returned to the cultivation system was minimal (Supplementary material, Table S4). During the recycling experiments, an increase in the $\mathrm{pH}$ of the culture medium (from 10 to 10.8) and a decrease in the inorganic carbon concentration (from $1798 \pm 0$ to $913 \pm 69 \mathrm{mg} \mathrm{L}^{-1}$ ) were observed as a result of the photosynthetic activity of the microalgae without $\mathrm{CO}_{2}$ addition (Table S4, Supplementary material). Flocculation did not affect the $\mathrm{pH}$, which is essential for effective biogas upgrading using microalgae.

Moreover, flocculation was uniform and was not selective to particular microalgal species of the consortium. As observed by microscopic analysis, no change in the microalgae community was found during any of the recycling experiments. Chlorella sp. and Oscillatoria sp. continuously dominated the consortium along with uncharacterized bacterial species. 

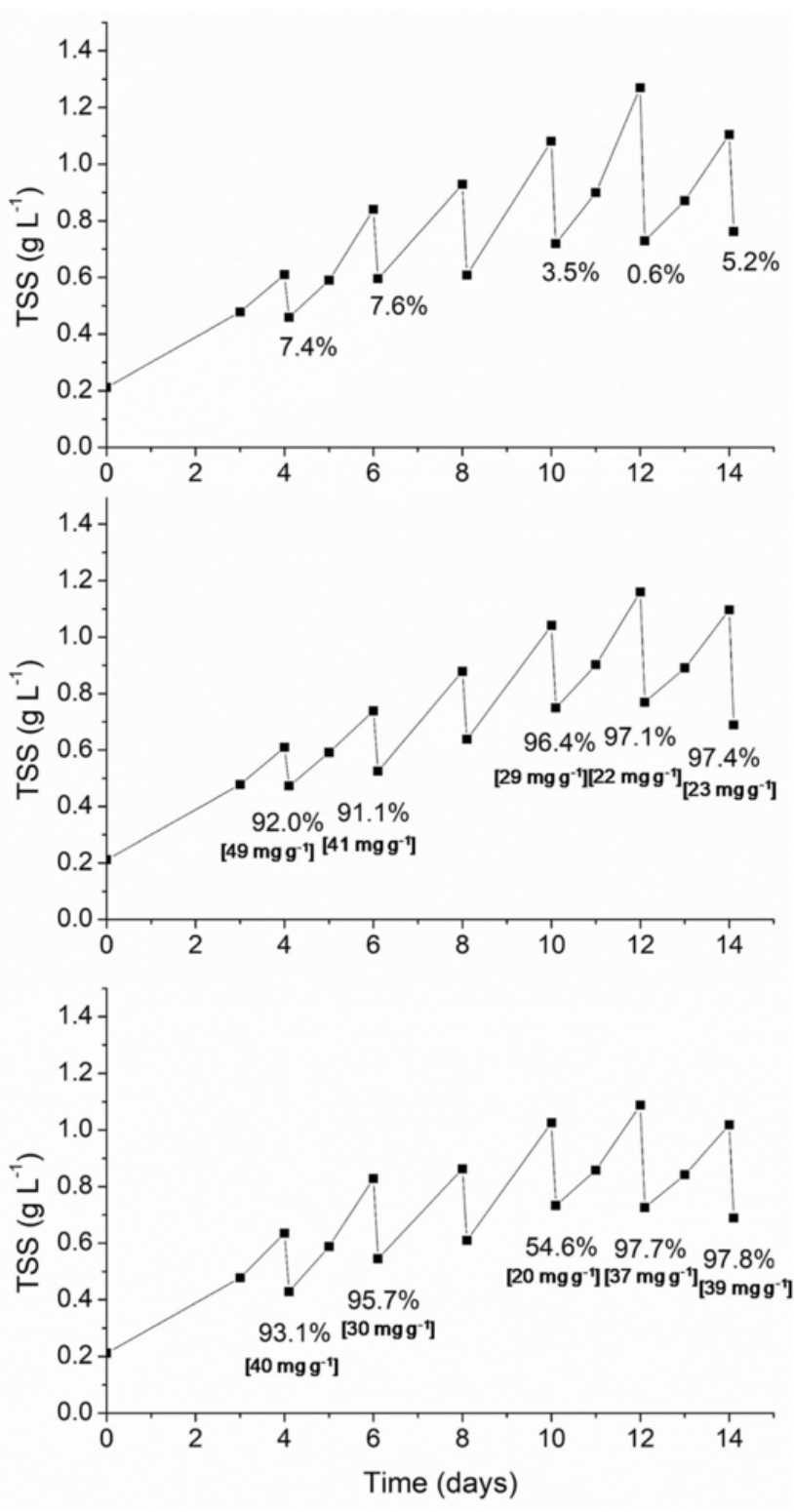

Fig. 2. Growth curve of the microalgal-bacterial consortium in the recycling medium with a) centrifugation (control) and flocculation with b) Zetag and c) cationic cellulose nanocrystals. The values below represent the flocculation efficiencies (\%) and dose of flocculants $\left(\mathrm{mg} \mathrm{g}^{-1}\right)$ during each harvesting cycle.

\subsection{Biomass separation after flocculation}

Following flocculation, separation of biomass flocs from the culture medium is an important process step. The biomass concentration factor is an indicator of the efficiency of biomass separation. Separation was achieved by gravity sedimentation of the flocs for $30 \mathrm{~min}$. The biomass concentration factor during repeated recycling experiments was lower than the ones observed during dose-response experiments (refer to Section 3.2.). Zetag-based flocculation resulted in concentration factors in the range of 3.2-7.7, whereas MIM- $g$-CNCs-based flocculation resulted in a maximum concentration factor of only 2.0 (Fig. 3; Supplementary material, Table S4). The higher concentration factors obtained for Zetag as the flocculant in comparison to MIM- $g$-CNCs could be attributed to a larger floc size and more compact structure as generated with the former (Fig. 3). In this context, Zhang et al. [45] proposed that not only the size of the flocs has influence on the settling velocity and the concentration factor of the microalgal biomass, but also the structure of these flocs, where microalgal flocs with large and compact structure should settle better under gravity.

In order to improve the concentration factor, screening was evaluated as a separation method. The biomass flocs obtained with Zetag and MIM-g-CNCs were allowed to settle for $30 \mathrm{~min}$ and screened through a nylon mesh screen with a pore size of $180 \mu \mathrm{m}$. Microalgal-bacterial culture without flocculants (acting as a control) resulted in harvesting efficiencies of $18 \%$ and $24 \%$ following 30 min settling and $180 \mu \mathrm{m}$ screening, respectively. The cell size of microalgae in this consortium varied between 0.5 and $200 \mu \mathrm{m}$. Without flocculation, most of the cells crossed the $180 \mu \mathrm{m}$ screen. In addition, a $30 \mu \mathrm{m}$ pore size screen was also tested, but this was not efficient due to clogging of the mesh. On the other hand, Zetag-based flocculation resulted in harvesting efficiencies of $97 \%$ for both, settling and $180 \mu \mathrm{m}$ screening. Similarly, MIM- $g$-CNCs-based flocculation resulted in harvesting efficiencies of $98 \%$ and $95 \%$ for settling and $180 \mu \mathrm{m}$ screening, respectively (Fig. 4). The slight lower harvesting efficiency for MIM- $g$-CNCs with a $180 \mu \mathrm{m}$ screen could be due to the fact that some smaller flocs or individual cells that were not flocculated passed through the screen. In this context, Verfaillie et al. [26] reported a low harvesting efficiency when using flocculation with cationically-modified CNCs followed by screening through a mesh with pore size of $180 \mu \mathrm{m}$ due to unstable structural integrity of the flocs.

Screening resulted in higher biomass concentration factors (up to 19.8; Fig. 4) compared to those for centrifugation (maximum value of 10; Supplementary material, Table S4). With Zetag-based flocculation, concentration factors of 3.7 and 17.7 were obtained for 30 min settling and $180 \mu \mathrm{m}$ screening, respectively. With MIM-g-CNCs-based flocculation, a concentration factor of 19.8 was obtained with screening. This value is $\sim 15$ times higher than the concentration factors obtained with gravity settling (1.3; Fig. 4). Hwang et al. [46] reported a maximum concentration factor of 25 using a cross-flow membrane filtration system of polyethylene terephthalate with a pore size of $4 \mu \mathrm{m}$ using a $3 \%$ of polyvinyl alcohol as coating material for harvesting Chlorella sp. Monte et al. [47] obtained a concentration factor of 4.8 with a loss of integrity of $10 \%$ while harvesting Dunaliella salina using a microfiltration membrane with a nominal pore size of $0.1 \mu \mathrm{m}$ made of polyethersulfone.

In spite of demanding slightly higher energy costs $\left(0.4 \mathrm{kWh} / \mathrm{m}^{3}\right.$ for screening vs $0.1 \mathrm{kWh} / \mathrm{m}^{3}$ for gravity settling) [48], considering the advantages of achieving a high biomass concentration in a short time, screening using a $180 \mu \mathrm{m}$ nylon mesh could be a good alternative to gravity sedimentation after flocculation.

\section{Conclusions}

In this study, five different flocculants were tested to harvest microalgal-bacterial biomass from a photosynthetic biogas upgrading process. Zetag and MIM- $g$-CNCs resulted in flocculation efficiencies $>92 \%$ at 30 and $20 \mathrm{mg} \mathrm{g}^{-1}$, respectively. Both flocculants were effective in harvesting biomass under semi-continuous cultivation with repeated recycling of spent medium. Moreover, both Zetag and MIM- $g$-CNCs did not result in any detrimental effect on either microalgal growth or $\mathrm{pH}$ of the spent medium during 5 cycles of harvesting. Finally, screening of the biomass flocs with a nylon mesh with $180 \mu \mathrm{m}$ pore size was demonstrated to achieve high biomass concentration factors. This flocculation-based harvesting is rapid and efficient in solid-liquid separation and hence could be applied in current biogas upgrading processes to replace the traditional gravity settlers-based harvesting.

\section{CRediT authorship contribution statement}

María del Rosario Rodero:Conceptualization, Investigation, Formal analysis, Writing - original draft, Writing - review \& editing.Raú 
a)

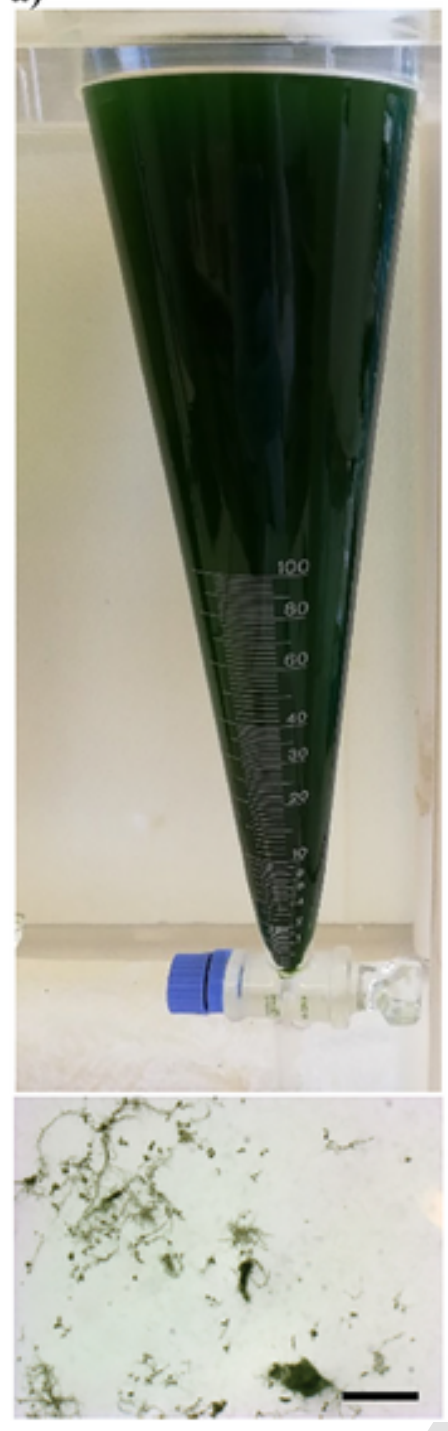

b)

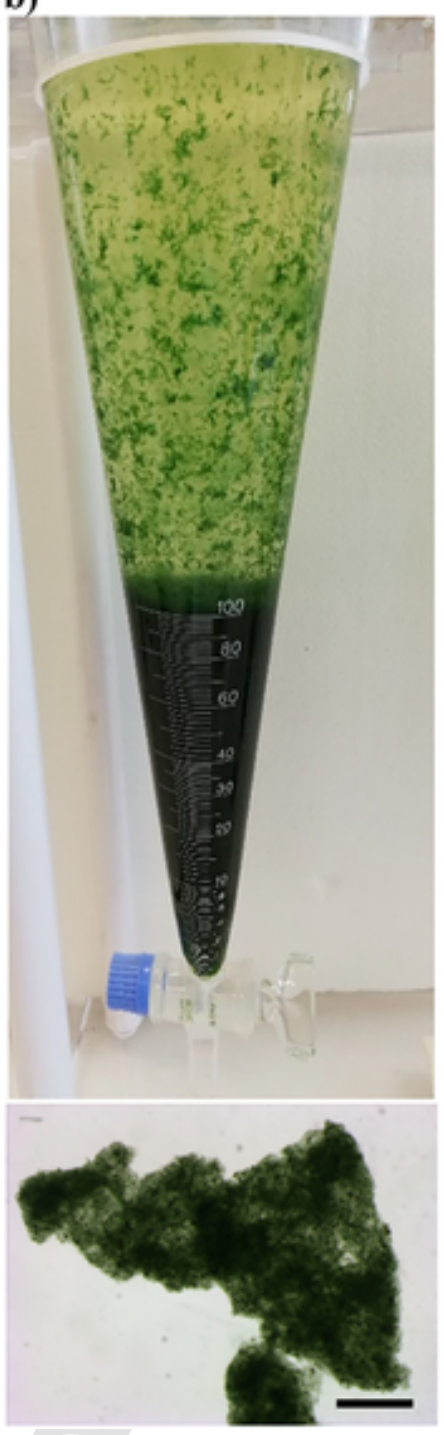

c)

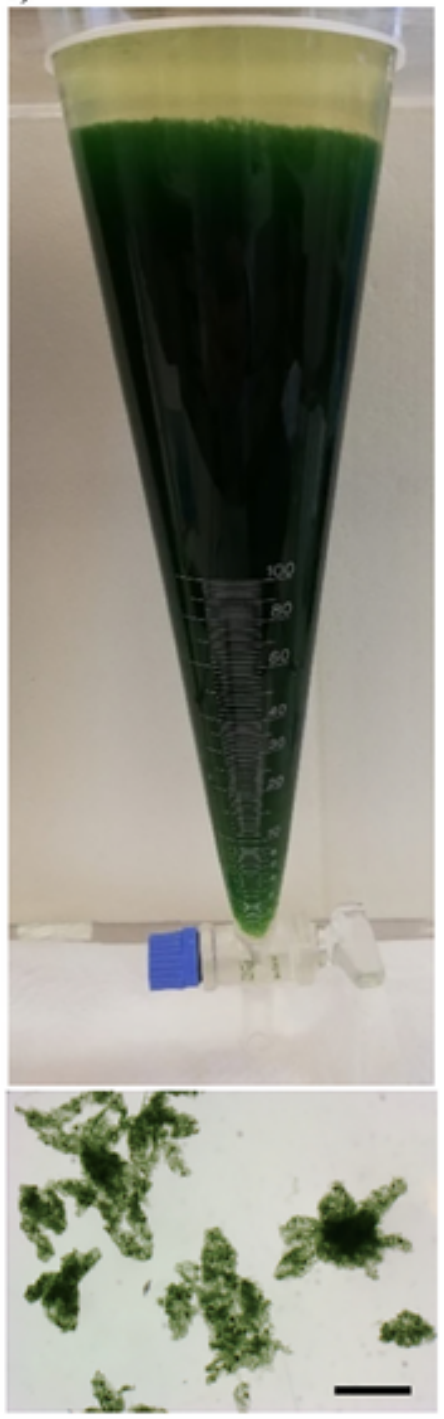

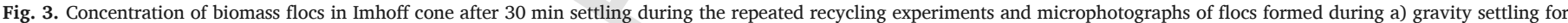
$30 \mathrm{~min}, \mathrm{~b})$ Zetag-based flocculation and c) cationic cellulose nanocrystals-based flocculation. Scale bar represents $250 \mu \mathrm{m}$.

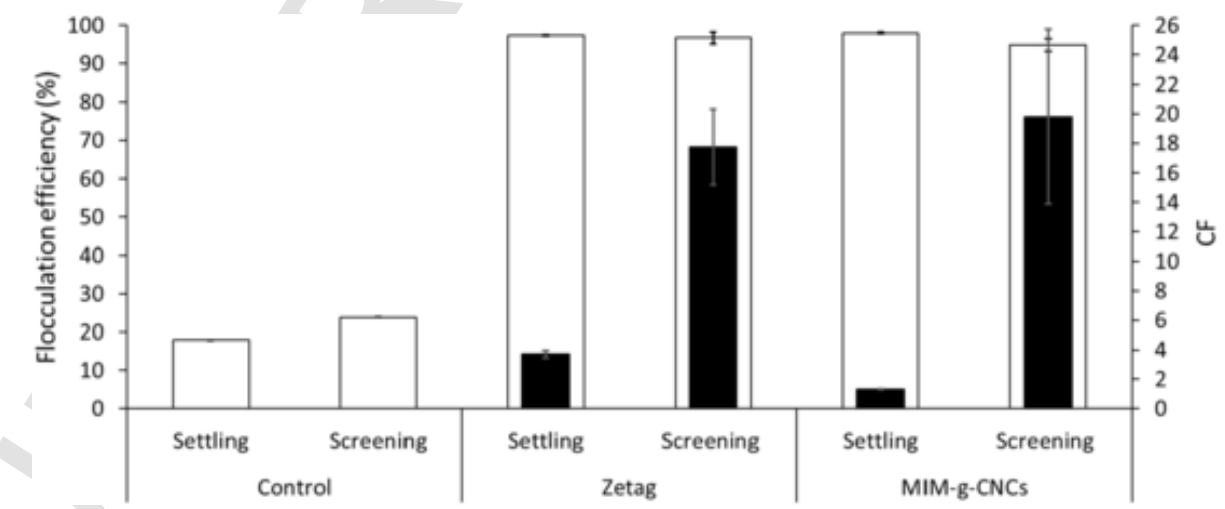

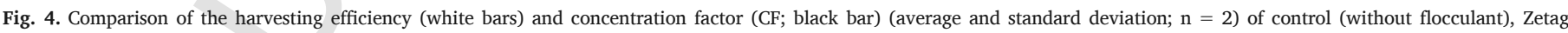

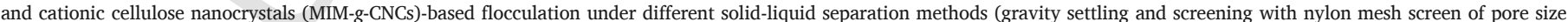
$180 \mu \mathrm{m})$.

1 Muñoz:Funding acquisition, Conceptualization, Writing - review \& editing.Raquel Lebrero:Funding acquisition, Conceptualization, Writing - review \& editing.An Verfaillie:Resources, Writing - review \& edit-
ing.Jonas Blockx:Resources, Writing - review \& editing.Wim Thielemans:Resources, Writing - review \& editing.Koenraad Muylaert:Funding acquisition, Conceptualization, Writing - review \& editing.Ra- 
masamy Praveenkumar:Conceptualization, Investigation, Formal analysis, Writing - original draft, Writing - review \& editing.

\section{Declaration of competing interest}

The authors declare that they have no known competing financial interests or personal relationships that could have appeared to influence the work reported in this paper.

\section{Acknowledgements}

This work was supported by the COST Action ES1408 European network for algal-bioproducts (EUALGAE), the Regional Government of Castilla y León and the EU-FEDER programme (CLU 2017-09 and UIC 071), and FWO-NRF cooperation project (VS00218N). Praveen Ramasamy received funding from the European Union's Horizon 2020 research and innovation programme under grant agreement no 751637. WT and KM thank FWO (grant G060816N) and Interreg Vlaanderen-Wallonie-France (ALPO project) for financial support.

\section{Statement of informed consent, human/animal rights}

No conflicts, informed consent, or human or animal rights are applicable to this study.

\section{Appendix A. Supplementary data}

Supplementary data to this article can be found online at https://doi. org/10.1016/j.algal.2020.101915.

\section{References}

[1] E Ryckebosch, M Drouillon, H Vervaeren, Techniques for transformation of biogas to biomethane, Biomass Bioenergy 35 (2011) 1633-1645, doi:10.1016/ j.biombioe.2011.02.033.

[2] R Muñoz, L Meier, I Díaz, D Jeison, A review on the state-of-the-art of physical/ chemical and biological technologies for biogas upgrading, Rev. Environ. Sci. Bio/ Technology (2015) 727-759, doi:10.1007/s11157-015-9379-1.

[3] A Toledo-Cervantes, J M Estrada, R Lebrero, R Muñoz, A comparative analysis of biogas upgrading technologies: photosynthetic vs physical/chemical processes, Algal Res. 25 (2017) 237-243, doi:10.1016/j.algal.2017.05.006.

[4] M del R Rodero, E Posadas, A Toledo-Cervantes, R Lebrero, R Muñoz, Influence of alkalinity and temperature on photosynthetic biogas upgrading efficiency in high rate algal ponds, Algal Res. 33 (2018) 284-290, doi:10.1016/j.algal.2018.06.001.

[5] A Bose, R Lin, K Rajendran, R O'Shea, A Xia, J D Murphy, How to optimise photosynthetic biogas upgrading: a perspective on system design and microalgae selection, Biotechnol. Adv. (2019), doi:10.1016/j.biotechadv.2019.107444 107444.

[6] E Posadas, D Marín, S Blanco, R Lebrero, R Muñoz, Simultaneous biogas upgrading and centrate treatment in an outdoors pilot scale high rate algal pond, Bioresour. Technol. 232 (2017) 133-141, doi:10.1016/j.biortech.2017.01.071.

[7] G Singh, S K Patidar, Microalgae harvesting techniques: a review, J. Environ. Manag. 217 (2018) 499-508, doi:10.1016/j.jenvman.2018.04.010.

[8] D H Cho, R Ramanan, J Heo, Z Kang, B H Kim, C Y Ahn, H M Oh, H S Kim, Organic carbon, influent microbial diversity and temperature strongly influence algal diversity and biomass in raceway ponds treating raw municipal wastewater, Bioresour. Technol. 191 (2015) 481-487, doi:10.1016/j.biortech.2015.02.013.

[9] M L Gerardo, S Van Den Hende, H Vervaeren, T Coward, S C Skill, Harvesting of microalgae within a biorefinery approach: a review of the developments and case studies from pilot-plants, Algal Res. 11 (2015) 248-262, doi:10.1016/ j.algal.2015.06.019.

[10] R Gutiérrez, F Passos, I Ferrer, E Uggetti, J García, Harvesting microalgae from wastewater treatment systems with natural flocculants: effect on biomass settling and biogas production, Algal Res. 9 (2015) 204-211, doi:10.1016/ j.algal.2015.03.010.

[11] L Zhu, Z Li, E Hiltunen, Microalgae Chlorella vulgaris biomass harvesting by natural flocculant: effects on biomass sedimentation, spent medium recycling and lipid extraction, Biotechnol. Biofuels 11 (2018) 1-10, doi:10.1186/ s13068-018-1183-z.

[12] D Vandamme, I Foubert, K Muylaert, Flocculation as a low-cost method for harvesting microalgae for bulk biomass production, Trends Biotechnol. 31 (2013) 233-239, doi:10.1016/j.tibtech.2012.12.005.

[13] F Roselet, D Vandamme, K Muylaert, P C Abreu, Harvesting of microalgae for biomass production, in: M A Alam, Z Wang (Eds.), Microalgae Biotechnol. Dev. Biofuel Wastewater Treat, Springer Singapore, Singapore, 2019, pp. 211-243, doi:10.1007/978-981-13-2264-8_10.
[14] A Augustine, A Tanwar, R Tremblay, S Kumar, Flocculation processes optimization for reuse of culture medium without $\mathrm{pH}$ neutralization, Algal Res. 39 (2019), doi:10.1016/j.algal.2019.101437 101437.

[15] I De Godos, H O Guzman, R Soto, P A García-encina, E Becares, R Muñoz, V A Vargas, Coagulation/flocculation-based removal of algal - bacterial biomass from piggery wastewater treatment, Bioresour. Technol. 102 (2011) 923-927, doi:10.1016/j.biortech.2010.09.036.

[16] I A Matter, V K Hoang Bui, M Jung, J Y Seo, Y E Kim, Y C Lee, Y K Oh, Flocculation harvesting techniques for microalgae: a review, Appl. Sci. 9 (2019), doi:10.3390/app9153069.

[17] G P 't Lam, M H Vermuë, G Olivieri, L A M van den Broek, M J Barbosa, M H M Eppink, R H Wijffels, D M M Kleinegris, Cationic polymers for successful flocculation of marine microalgae, Bioresour. Technol. 169 (2014) 804-807, doi:10.1016/j.biortech.2014.07.070.

[18] F Roselet, D Vandamme, M Roselet, K Muylaert, P C Abreu, Screening of commercial natural and synthetic cationic polymers for flocculation of freshwater and marine microalgae and effects of molecular weight and charge density, Algal Res. 10 (2015) 183-188, doi:10.1016/j.algal.2015.05.008.

[19] N Rashid, S Ur, J Han, Rapid harvesting of freshwater microalgae using chitosan, Process Biochem. 48 (2013) 1107-1110, doi:10.1016/j.procbio.2013.04.018.

[20] J Blockx, A Verfaillie, W Thielemans, K Muylaert, Unravelling the mechanism of chitosan-driven flocculation of microalgae in seawater as a function of $\mathrm{pH}, \mathrm{ACS}$ Sustain. Chem. Eng. 6 (2018) 11273-11279, doi:10.1021/ acssuschemeng.7b04802.

[21] M S Farid, A Shariati, A Badakhshan, B Anvaripour, Using nano-chitosan for harvesting microalga Nannochloropsis sp., Bioresour. Technol. 131 (2013) 555-559, doi:10.1016/j.biortech.2013.01.058.

[22] M M Barrado-Moreno, J Beltrán-Heredia, J Martín-Gallardo, Microalgal removal with natural coagulants, Phycologia 55 (2016) 688-695, doi:10.2216/15-113.1.

[23] D Vandamme, S Eyley, G Van Den Mooter, K Muylaert, W Thielemans, Highly charged cellulose-based nanocrystals as flocculants for harvesting Chlorella vulgaris, Bioresour. Technol. 194 (2015) 270-275, doi:10.1016/ j.biortech.2015.07.039

[24] S Eyley, D Vandamme, S Lama, G Van Den Mooter, K Muylaert, W Thielemans, $\mathrm{CO}_{2}$ controlled flocculation of microalgae using $\mathrm{pH}$ responsive cellulose nanocrystals, 7, Nanoscale, 2015, pp. 14413-14421.

[25] J Blockx, A Verfaillie, S Eyley, O Deschaume, C Bartic, K Muylaert, W Thielemans, Cationic cellulose nanocrystals for flocculation of microalgae: effect of degree of substitution and crystallinity, ACS Appl. Nano Mater. 2 (2019) 3394-3403, doi:10.1021/acsanm.9b00315.

[26] A Verfaillie, J Blockx, R Praveenkumar, W Thielemans, K Muylaert, Harvesting of marine microalgae using cationic cellulose nanocrystals, Carbohydr. Polym. (2020), doi:10.1016/j.carbpol.2020.116165 116165 In press.

[27] Y Habibi, L A Lucia, O J Rojas, Cellulose Nanocrystals: Chemistry, Self-assembly, and Applications, 110, 2010, pp. 3479-3500.

[28] S Eyley, W Thielemans, Surface modification of cellulose nanocrystals, Nanoscale 6 (2014) 7764-7779, doi:10.1039/C4NR01756K

[29] F Roselet, D Vandamme, M Roselet, K Muylaert, P C Abreu, Effects of pH, Salinity, Biomass Concentration, and Algal Organic Matter on Flocculant Efficiency of Synthetic Versus Natural Polymers for Harvesting Microalgae Biomass, 10 , Bioenerg. Res. , 2017, pp. 427-437.

[30] D Bilanovic, G Shelef, A Sukenik, Flocculation of microalgae with cationic polymers - effects of medium salinity, Biomass 17 (1988) 65-76, doi:10.1016/ 0144-4565(88)90071-6.

[31] G P Lam, J B Giraldo, M H Vermuë, G Olivieri, M H M Eppink, R H Wijffels, Understanding the salinity effect on cationic polymers in inducing flocculation of the microalga Neochloris oleoabundans, J. Biotechnol. 225 (2016) 10-17, doi:10.1016/j.jbiotec.2016.03.009.

[32] D Marín, E Posadas, P Cano, V Pérez, S Blanco, R Lebrero, R Muñoz, Seasonal variation of biogas upgrading coupled with digestate treatment in an outdoors pilot scale algal-bacterial photobioreactor, Bioresour. Technol. 263 (2018) 58-66, doi:10.1016/j.biortech.2018.04.117

[33] L Rodolfi, G C Zittelli, L Barsanti, G Rosati, M R Tredici, Growth medium recycling in Nannochloropsis sp. mass cultivation, Biomol. Eng. 20 (2003) 243-248, doi:10.1016/S1389-0344(03)00063-7.

[34] S Fon Sing, A Isdepsky, M A Borowitzka, D M Lewis, Pilot-scale continuous recycling of growth medium for the mass culture of a halotolerant Tetraselmis sp. in raceway ponds under increasing salinity: a novel protocol for commercial microalgal biomass production, Bioresour. Technol. 161 (2014) 47-54, doi:10.1016/j.biortech.2014.03.010.

[35] R R L Guillard, C J Lorenzen, Yellow-green algae with chlorophyllide C1,2, J. Phycol. 8 (1972) 10-14, doi:10.1111/j.1529-8817.1972.tb03995.x.

[36] M del R Rodero, A Carvajal, V Castro, D Navia, C de Prada, R Lebrero, R Muñoz, Development of a control strategy to cope with biogas flowrate variations during photosynthetic biogas upgrading, Biomass Bioenergy 131 (2019), doi:10.1016/ j.biombioe.2019.105414

[37] A D Eaton, L S Clesceri, E W Rice, A E Greenberg, M A H Franson, in: Centen (Ed.), APHA: Standard Methods for the Examination of Water and Wastewater, APHA, AWWA, WEF, Washington, DC, 2005.

[38] Z T Harith, F M Yusoff, M S Mohamed, M Shariff, M Din, A B Ariff, Effect of different flocculants on the flocculation performance of microalgae, Chaetoceros calcitrans, Cells 8 (2009) 5971-5978.

[39] F Delrue, Y Imbert, G Fleury, G Peltier, J-F Sassi, Using coagulation-flocculation to harvest Chlamydomonas reinhardtii: coagulant and flocculant efficiencies, and reuse of the liquid phase as growth medium, Algal Res. 9 (2015) 283-290, doi:10.1016/j.algal.2015.04.004.

[40] J-W Yang, A Shrivastav, W Farooq, S K Mishra, W I Suh, B Ryu, M Moon, M S Park, Effect of harvesting methods on the reusability of water for cultivation of Chlorella vulgaris, its lipid productivity and biodiesel quality, Algal Res. 8 (2015) 1-7, doi:10.1016/j.algal.2014.12.007. 
[41] N F H Selesu, T V. de Oliveira, D O Corrêa, B Miyawaki, A B Mariano, J V C Vargas, R B Vieira, Maximum microalgae biomass harvesting via flocculation in large scale photobioreactor cultivation, Can. J. Chem. Eng. 94 (2016) 304-309, doi:10.1002/cjce.22391.

[42] E Molina Grima, E H Belarbi, F G Acién Fernández, A Robles Medina, Y Chisti, Recovery of microalgal biomass and metabolites: process options and economics, Biotechnol. Adv. 20 (2003) 491-515, doi:10.1016/S0734-9750(02)00050-2.

[43] Y Gerchman, B Vasker, M Tavasi, Y Mishael, Y Kinel-Tahan, Y Yehoshua, Effective harvesting of microalgae: comparison of different polymeric flocculants, Bioresour. Technol. 228 (2017) 141-146, doi:10.1016/j.biortech.2016.12.040.

[44] S Lama, K Muylaert, T Bahadur, I Foubert, R K Henderson, D Vandamme, Flocculation properties of several microalgae and a cyanobacterium species during ferric chloride, chitosan and alkaline flocculation, Bioresour. Technol. 220 (2016) 464-470, doi:10.1016/j.biortech.2016.08.080.
[45] H Zhang, L Yang, X Zang, S Cheng, X Zhang, Effect of shear rate on floc characteristics and concentration factors for the harvesting of Chlorella vulgaris using coagulation-flocculation-sedimentation, Sci. Total Environ. 688 (2019) 811-817, doi:10.1016/j.scitotenv.2019.06.321.

[46] T Hwang, S J Park, Y K Oh, N Rashid, J I Han, Harvesting of Chlorella sp. KR-1 using a cross-flow membrane filtration system equipped with an anti-fouling membrane, Bioresour. Technol. 139 (2013) 379-382, doi:10.1016/ j.biortech.2013.03.149.

[47] J Monte, M Sá, C F Galinha, L Costa, H Hoekstra, C Brazinha, J G Crespo, Harvesting of Dunaliella salina by membrane filtration at pilot scale, Sep. Purif. Technol. 190 (2018) 252-260, doi:10.1016/j.seppur.2017.08.019.

[48] N Uduman, Y Qi, M K Danquah, G M Forde, A Hoadley, Dewatering of microalgal cultures: a major bottleneck to algae-based fuels, J. Renew. Sustain. Energy 2 (2010), doi:10.1063/1.3294480. 